\title{
THERMAL OSCILLATIIONS IN LIQUID HELIUM TARGETS
}

\author{
L. Wang and L.X. Jia \\ Brookhaven National Laboratory \\ Upton, New York 11973, USA
}

\begin{abstract}
A liquid helium target for the high-energy physics was built and installed in the proton beam line at the Alternate Gradient Synchrotron of Brookhaven National Laboratory in 2001. The target flask has a liquid volume of 8.25 liters and is made of thin Mylar film. A G-M/J-T cryocooler of five-watts at $4.2 \mathrm{~K}$ was used to produce liquid helium and refrigerate the target. A thermosyphon circuit for the target was connected to the J-T circuit by a liquid/gas separator. Because of the large heat load to the target and its long transfer lines, thermal oscillations were observed during the system tests. To eliminate the oscillation, a series of tests and analyses were carried out. This paper describes the phenomena and provides the understanding of the thermal oscillations in the target system.
\end{abstract}

\section{INTR.ODUCTION}

Cryogen targets have been used in the experiments of the high-energy physics research for more than 35 years at Brookhaven National Laboratory. Hundreds of targets involving liquid helium3, helium4, hydrogen, and deuterium have been built and installed in the proton beam lines at the Alternate Gradient Synchrotron of BNL. Although various refrigeration methods have been applied to the cryogen targets according to the physics requirements, the thermosyphon flow circuit is commonly adopted to transfer the cryogen to the target [1-2]. The thermosyphon flow always involves two-phase cryogen flow. Unlike the forced two-phase flow, the mass flow rate and the pressure differential in the thermosyphon system are not the operating parameters, since the flow is driven by the gravity potential. The liquid flasks of the targets in various shapes are often made of thin Mylar films in tenths of millimeter, and positioned in vacuum chambers that normally consist of one or two Mylar film windows at up stream or down stream of the particlebeam line [3]. Because of the sensitivity of the particle counters, the thermal insulation materials are strictly limited surrounding the liquid flask, which result in large radiation heat load towards the target.

Thermal oscillation in targets is one of the crucial concerns in cryogenic system design. The phenomena have been observed in target systems operated under certain conditions. The temperatures at the supply and return lines of the target could oscillate at very large amplitude above the critical temperature. The corresponding pressure oscillation in the target could severely disturb the liquid helium pool in the target. Sometimes it caused 
the entire system to oscillate. The instability of the fluid in the target presents serious problem to the physics.

Thermal oscillation is mostly brought about by the large heat load to the Mylar target. It is also the nature of thermosyphon driven flow with long transfer lines, which induces the heat leak to the flow. It is almost certain to happen when the refrigeration is marginal to the heat load of the target. The system instability caused by this kind of oscillation could stop the cooling of the target even though the refrigeration is slightly higher than the heat load. The following sections discuss the phenomena and provide the analysis on thermal oscillation in a liquid helivum target.

\section{LIQUTD HELIUM TARGET SYSTEM}

The liquid helium target system was built for physics experiment of E931 at AGS in 1999 [4-5]. The target was refrigerated by a G-M/J-T cryocooler. The Mylar flask has a liquid volume of 8.25 liters. The total heat load is 3 watts against the refrigerator, which includes the thermal radiation to the target flask of 1.85 watts, plus another 1.15 watts introduced by the cryo-valve stems, transfer lines and liquid/gas separator, etc. The G-M/J$\mathrm{T}$ cryocooler built has a maximum cooling capacity of 5 watts. The G$\mathrm{M} / \mathrm{J}-\mathrm{T}$ flow runs at a mass flow rate of $0.8 \sim 1.0 \mathrm{~g} / \mathrm{s}$ at $16 \mathrm{~atm}$ pressure differential. The thermosyphon flow circuit for the target is connected to the J-T flow circuit at the liquid/gas separator. The system flow diagram is given in FIGURE 1. The J-T flow circuit can be operated without involving the target once the two isolation cryo-valves are closed for the target supply and return. When the $\mathrm{J}-\mathrm{T}$ flash starts in the separator, the target precooling can be accomplished by opening the supply valve and bypass valve allowing the warm gas from the target flows directly to the compressor. The thermosyphon flow circuit is established when the cryovalves are open. The liquid helium flows down from the bottom of the separator into the flask at the bottom. Vapor helium flows up from the top of the target into separator at top, where it joins the vapor firaction of J-T flow back to the heat exchanger train.

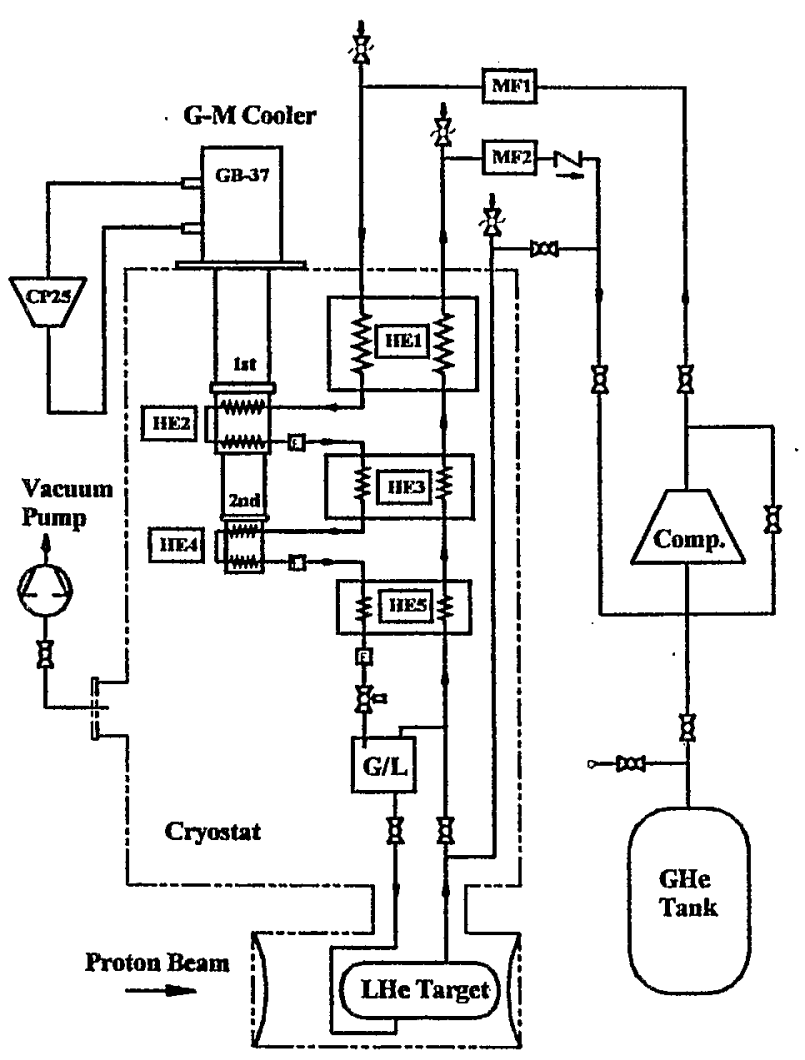

FIGURE 1. System flow diagram. 


\section{THERMOSYPHON ANALYSIS}

The typical thermosyphon flow circuit in a target system is depicted in FIGURE 2. A set of Bernoulli's equations governing the equilibrium homogeneous two-phase flow is given as follows. The major assumption is that the mass flow rates in supply and return lines are balanced, which imply a stable liquid level in the target [1].

$$
\begin{gathered}
\left(p_{i}+\frac{1}{2} \rho_{i} v_{i}^{2}+g \rho_{i} L_{i}\right)-\left(p_{i+1}+\frac{1}{2} \rho_{i+1} v_{i+1}^{2}+g \rho_{i+1} L_{i+1}\right)-\frac{4\left(L_{i}-L_{i+1}\right)}{D_{i+1}} \frac{1}{2} \bar{\rho}_{i+1} v_{i+1}^{2} \bar{f}_{i+1}=0 \\
v_{i}-\frac{4 \dot{m}}{\rho_{i} \pi D_{i}^{2}}=0 \\
\bar{f}_{i}-0.079\left(\frac{4 \dot{m}}{\pi \bar{\mu}_{i} D_{i}}\right)^{-0.25}=0 \\
\bar{\rho}_{M}-\left[\left(1-\frac{Q}{\dot{m} h_{f}}\right) \rho_{L}+\frac{Q}{\dot{m} h_{f}} \rho_{G}\right]=0 \\
\bar{\mu}_{M}-\left[\left(1-\frac{Q}{\dot{m} h_{f}}\right) \mu_{L}+\frac{Q}{\dot{m} h_{f}} \mu_{G}\right]=0
\end{gathered}
$$

Where, $p$ represents the static pressure at certain point; $\rho$ the fluid density; $v$ the velocity; $L$ and $D$ the length diameter of the flow passage; $\dot{m}$ the mass flow rate; $\bar{\rho}$ and $\bar{\mu}$ the average density and average viscosity of flow; $\bar{f}$ the average friction factor between the two calculation points; $Q$ the heat load to the target; $h_{f}$ the latent heat of fluid. The subscripts, $M, L$, and $G$, represent the two-phase, the liquid phase, and the vapor phase, respectively.

\section{THERMAL OSCILLATION}

Gravity differential is the driving potential of thermosyphon flow. The vapor pressure inside the separator is the lowest one in the circuit and is common for each branch of the flow. The lowest gravity potential is at the bottom of the target, where is also the separation point for the two

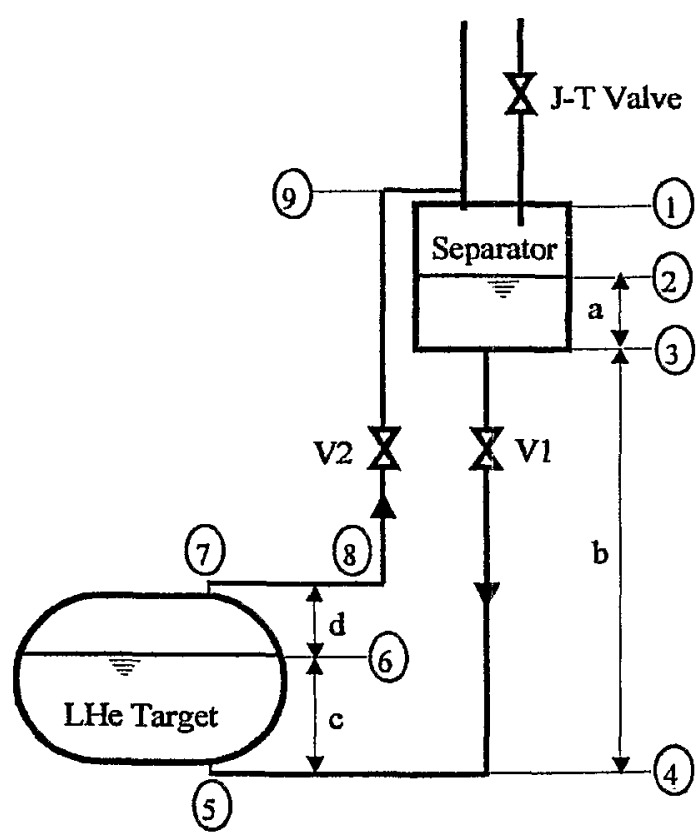

FIGURE 2. Typical thermosyphon flow circuit in target system. 
branches of the "U-tube". When the liquid level is established in the separator, the fluid density in the supply line is larger than that in the return line. Assuming the heat load is uniformly distributed on all cold surfaces, as normally does, a thermosyphon flow could be established eventually as long as liquid level in the separator maintains. However, this positive balanced flow can be demolished under certain circumstances.

The mass flow rates in the supply and return lines in a thermosyphon circuit are not always balanced, especially during the condensing period. The un-balanced mass flow rates indicate the accumulation of liquid in the target. If the liquid gradually accumulates in the target, the mass flow rate in the return line is less than that in the supply line. The target behaves like a buffer vessel taking mass from the supply line and adjusting the liquid level accordingly. The supply flow is primarily up to the operating condition of J-T circuit provided that the cryo-valves are fully opened. However, the fluid in the return line is the vapor from the target, which is dominated by the heat load towards the target. Since the heat load to the target will not change with fluid flow, this implies that there is a characteristic mass flow rate in the return line. It can be expressed as $m_{c}=q_{t} / h_{f}$, where $q_{t}$ is the heat load to the target and $h_{f}$ is the latent heat of fluid. If the liquid producing rate in the $\mathrm{J}$-T flash is smaller than the characteristic mass flow rate, $m_{c}$, the liquid level in the target will drop. Any reduction of J-T refrigeration, if the target still contains liquid, could slow down the flow in the supply line. Therefore, the fluid quality in the supply line increases, and, eventually, the positive flow could be stopped. In the mean time, the vapor flow continues in the return line and the density of the fluid maintains at nearly constant for the existing heat load.

Assuming the homogenous flow and ignoring the friction resistance in both lines, a characteristic density in supply line, $\rho_{c}=\left(L_{t} \rho_{t}+L_{r} \rho_{r}\right) / L_{s}$ is defined, where $L_{t}, L_{r}$, and $L_{s}$ are, respectively, the gravitational heights of fluid in the target, return line, and supply line, and $\rho_{\mathrm{t}}, \rho_{\mathrm{r}}$ are the densities of the fluid in the target and return line. Using the characteristic mass flow rate, and the conderising or evaporating rates in the target, one can calculate the mass flow rate in the supply line. It can be written as $\dot{m}_{s}=\dot{m}_{r}+\Delta \dot{m}_{t}$, where $\dot{m}_{s}$ and $\dot{m}_{r}$ are the mass flow rates in the supply and return lines, and $\Delta \dot{m}_{t}$ is the mass change rate in the target. The result can be verified by the flashing rate at the J-T valve.

The heat load to the long supply lines will worsen the situation once the flow is stopped. The reduction of the mass flow rate in the supply line could result in reducing the density of fluid. When $\rho_{s}<\rho_{c}=\left(L_{t} \rho_{t}+L_{r} \rho_{r}\right) / L_{s}$, the positive flow stops. Meanwhile, and the denser fluid in the supply line is gradually formed. Then the positive flow rebuilds and the temperature along the supply line drops. The recovery of the positive flow could take a long time if the refrigeration is marginal. Using the temperature in this warm gas pot and heat load along the supply line, one can also calculate the corresponding recovery time at the given refrigeration.

The thermal oscillations are well illustrated in FIGURE 3 to 7, which show the temperature vs. time at the supply and return lines of the target. FIGURE 8 shows the pressure oscillation. The descriptions of each case are given in the following sections.

The case $I$ is shown in FIGURE 3. The target was 50\% full. The mass flow rate in J-T circuit was high as $1.06 \mathrm{~g} / \mathrm{s}$ at the beginning. Since the mass flow rate was higher than that of the maximum refrigeration required, the system refrigeration capacity dropped to an extent that the oscillation occurs. When it was reduced to $1.04 \mathrm{~g} / \mathrm{s}$ by adjusting the J-T valve, the refrigeration increased, and the amplitude of oscillation reduced.

The case II is shown in FIGURE 4. The target was $90 \%$ full at the time. The two valves in the transfer lines were fully opened. The mass flow rate was adjusted to $0.8 \mathrm{~g} / \mathrm{s}$. 


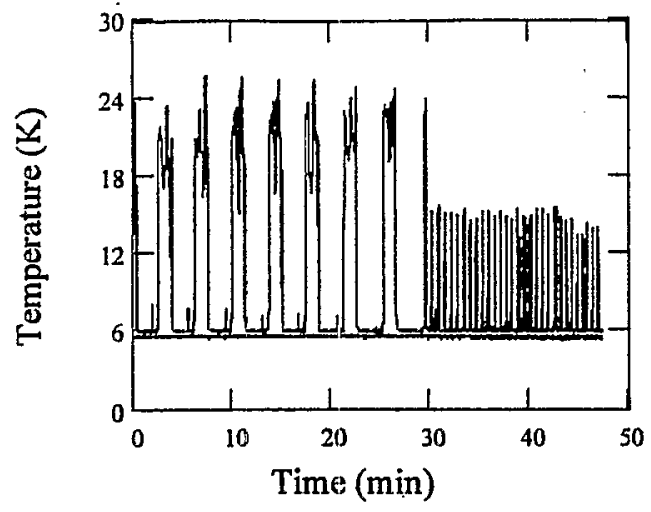

FIGURE 3. Target $50 \%$ full, $\mathrm{m}=1.04 \sim 1.06$ $\mathrm{g} / \mathrm{s}, \mathrm{V} 1$ and $\mathrm{V} 2$ fully open.

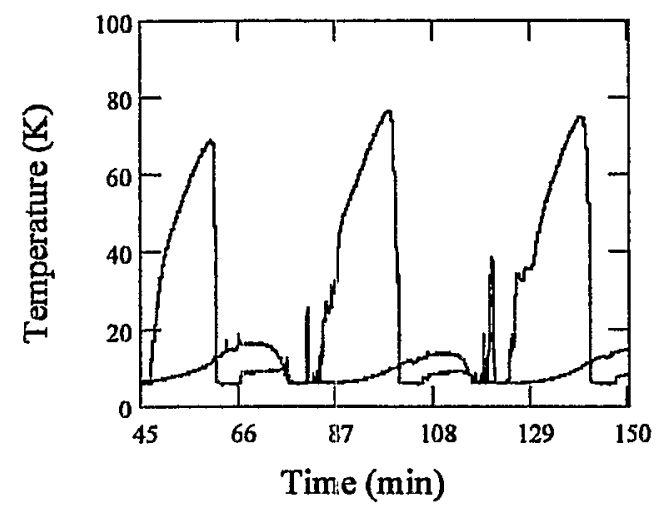

FIGURE 5. Target $10 \%$ full, $m<0.75 \mathrm{~g} / \mathrm{s}, \mathrm{V} 1$ partly open, V2 fully open.

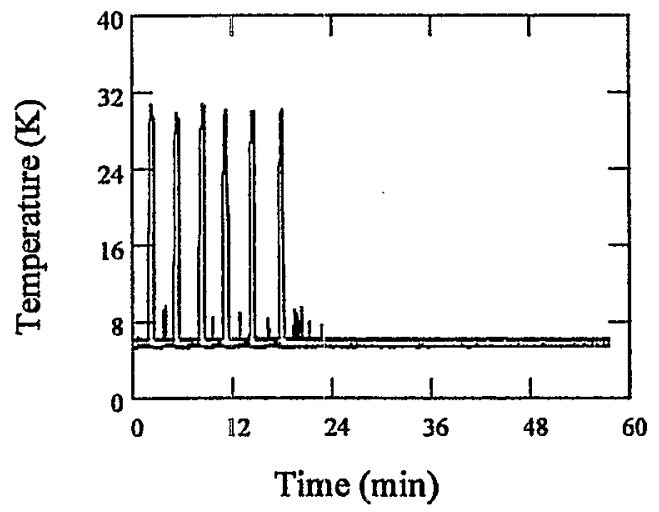

FIGURE 7. Target $90 \%$ full, $\mathrm{m}=0.7 \sim 0.85$ $\mathrm{g} / \mathrm{s}, \mathrm{V} 1$ and V2 fully open.

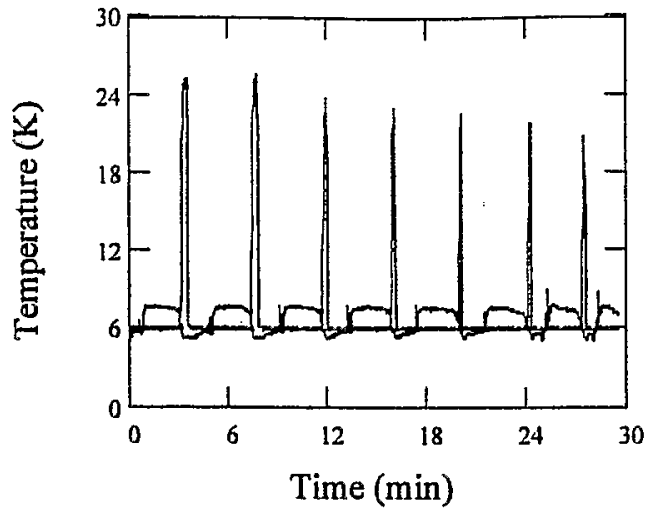

FIGURE 4. Target $90 \%$ full, $\mathrm{m}=0.8 \mathrm{~g} / \mathrm{s}, \mathrm{V} 1$ and V2 fully open.

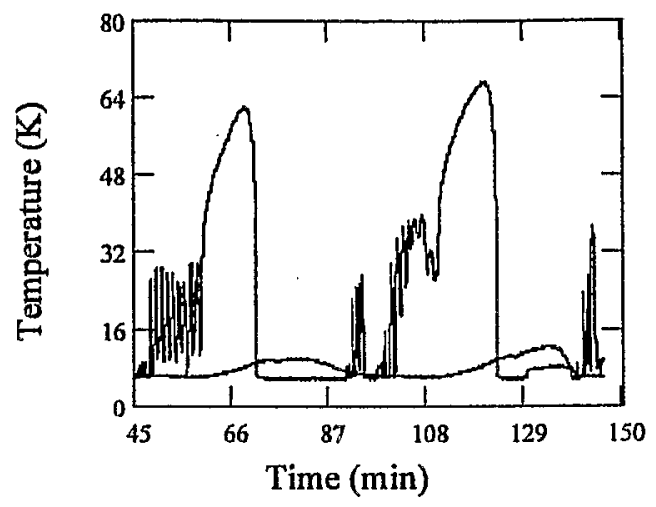

FIGURE 6. Target $30 \%$ full, $\mathrm{m}>0.75 \mathrm{~g} / \mathrm{s}$, V1 partly open, V2 fully open.

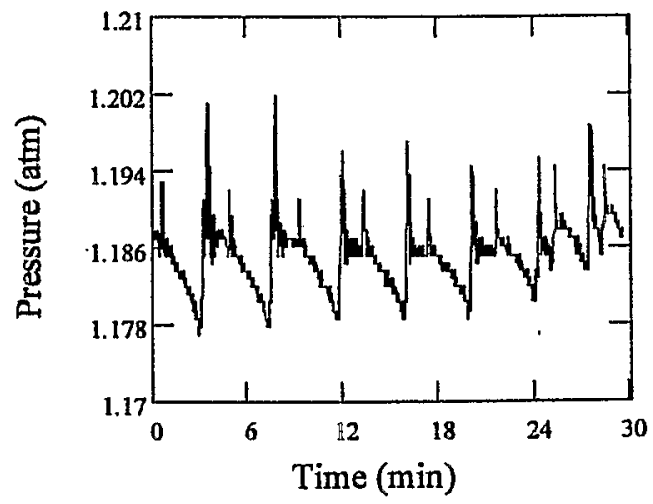

FIGURE 8. Target $90 \%$ full, $m=0.8 \mathrm{~g} / \mathrm{s}, \mathrm{V} 1$ and $\mathrm{V} 2$ fully open.

The oscillation occurred because the refrigeration was not high enough to match the heat load to the target.

The case III is shown in FIGURE 5. The target was about $10 \%$ full. The valve in the supply line was partly opened. The oscillation occurred, because the valve restricted the flow through the thermosyphon circuit. 
The case IV is shown in FIGURE 6. It was the same as the case III except that the mass flow rate was slightly higher than $0.75 \mathrm{~g} / \mathrm{s}$. And then the frequency of oscillation was reduced.

The case $\mathrm{V}$ is shown in FIGURE 7. Target was $90 \%$ full and the valves were both fully opened. The oscillation happened when the mass flow rate was $0.78 \mathrm{~g} / \mathrm{s}$, and then stopped when it increased to $0.85 \mathrm{~g} / \mathrm{s}$. No oscillation was observed when the mass flow rate was between 0.85 to $1.0 \mathrm{~g} / \mathrm{s}$. The system was running well and stable.

\section{CONCLUSION}

The thermosyphon flow in the target system described above is different from the one in the dewar filling system. The latter has a large liquid service dewar sitting at above the target, which has unlimited cooling power against the heat load at the target. As long as the service dewar contains enough liquid, the thermosyphon flow maintains stable. However, most of cryocoolers have limited cooling power for liquid helium target at $4.2 \mathrm{~K}$. The large target or large heat load requires large cryocooler for cooling capacity. Refrigeration budget for the separator-type target should be more abundant than the condenser-type one. The condenser-type targets are often used for liquid hydrogen and deuterium. In most liquid hydrogen and deuterium target systems the thermal oscillation does not present as the problem as that in the liquid helium target that uses cryocoolers, because most of the cryocoolers have high cooling capacity at $20 \mathrm{~K}$. Marginal refrigeration budget is one of the causes to thermal oscillation in the target systems. If the size of liquid helium target is larger than 10 liters, equivalent to a heat load of $4 \sim 5$ watts at $4.2 \mathrm{~K}$, the cryocoolers, such as $\mathrm{G}-\mathrm{M}$, pulse tube, and $\mathrm{G}-\mathrm{M} / \mathrm{J}-\mathrm{T}$, may not be the options.

To eliminate the thermal oscillation, the transfer lines in the thermosyphon circuit should avoid any adverse loops. The under sized transfer lines should also be avoided. Any thermal short, such as those existed in the short-stem cryo-valves and relief valves should be avoided or properly cold-intercepted.

Thermal oscillation described above is different from thermal acoustic oscillation that is oftentimes induced and amplified by pressure waves. The thermal oscillation in the thermosyphon flow is dominated and amplified by heat waves. The latter is featured in much lower frequency and less influenced by the mechanical structure than that in acoustic oscillations.

\section{ACKNOWLEDGEMENT}

This work is performed under the contract with the US Department of Energy.

\section{REFERENCES}

1. Jia, L. X., et al, "Safety Design, Operation, and Control of a Liquid Hydrogen Target at BNL", in Advances in Cryogenic Engineering 43A, edited by P. Kittel, Plenum Press, New York, 1998, pp. 629636.

2. Vail'ev, L. M., et al, "A Universal Liquid Hydrogen Target for Experiments with High Energy Particles", Cryogenics, 10:495 (1973).

3. Vail'ev, L. M., et al, "A Mylar Liquid Hydrogen Target with Thin Mylar Side Walls", Cryogenics, 12:387 (1972).

4. Jia, L. X., E931 LHe Target Design Manual, Brookhaven National Laboratory, 1999.

5. Jia, L. X., A Five-Watts G-M/J-T Refrigerator For LHe Target At BNL, paper presented at CEC/ICMC'01, Madison, WI, 2001. 